\title{
Bifurcations of optimal vector fields in the shallow lake model
}

\author{
Tatiana Kiseleva $^{* a}$, Florian Wagener ${ }^{a b}$ \\ ${ }^{a}$ CeNDEF, Department of Quantitative Economics, Universiteit van \\ Amsterdam, Roetersstraat 11, 1018 WB Amsterdam, The Nether- \\ lands. \\ ${ }^{b}$ Tinbergen Institute, Roetersstraat 31, 1018 WB Amsterdam, The \\ Netherlands. \\ Email: T.Kiseleva@uva.nl (Kiseleva) and F.O.O.Wagener@uva.nl \\ (Wagener). \\ * Corresponding author. T: +3120 5254214
}

\begin{abstract}
The solution structure of the set of optimal solutions of the shallow lake problem, a problem of optimal pollution management, is studied as we vary the values of the system parameters: the natural resilience, the relative importance of the resource for social welfare and the future discount rate. We find parameter values at which qualitative changes occur. Using theoretical results on the bifurcations of the solution structure to infinite horizon optimization problems obtained earlier, we give a fairly complete bifurcation analysis of the shallow lake problem. In particular, we show how the increase of the discount rate affects the parameter regions where an oligotrophic steady state, corresponding to low pollution level, is globally stable or locally stable under optimal dynamics. Asymptotically, an increase of the discount rate can be offset with a proportional increase of the relative social weight of the resource.
\end{abstract}

Key words: Optimal vector fields; Indifference points; Bifurcations;

JEL: C61, Q57

\section{Introduction}

A wide class of one state optimal control problems are non-convex dynamic optimization problems with parameters. Often in such problems there exist multiple equilibria, that may occur or disappear as the parameters vary. Among those equilibria more than one can be optimal to converge to, depending on an initial state of a system; it is also possible that none of the optimal paths cover the state space completely. Therefore such dynamic optimization problems may feature indifference states, which are initial states to more than one optimal 
solution. The difficulties with existence of multiple equilibria can make solving non-convex optimal control problems quite complicated.

In the present paper ideas from bifurcation theory, developed in Kiseleva and Wagener (in prep.), are used to analyze the effects of varying parameters in the shallow lake pollution problem, introduced in Brock and Starret (2003); Mäler et al (2003). This is an optimal pollution management problem where a social planner faces a trade-off between interests of farmers, who indirectly benefit from polluting the lake by using fertilizers that are washed into it, and interests of fishermen, tourists and water companies, who benefit from high quality of the lake water. The shallow lake model contains three parameters: $b$, the rate of loss of pollutant due to sedimentation, representing biological properties of the lake; $c$, the relative costs of pollution, modeling the trade-off between farmers' and tourists' interests; $\rho$, the discount rate, representing the intertemporal rate of substitution. The main idea of the bifurcation analysis is to study dependence of the solution structure upon the parameters of the model. Nonconvexities in this model exhibiting for some regions of the parameter values cause the existence of multiple local optima of the water pollution level and thereby history-dependent optimal pollution policies, see Brock and Starret (2003); Mäler et al (2003). In Wagener (2003) the genesis of history-dependent optimal management policies in the shallow lake model has been connected to the occurrence of heteroclinic bifurcations of the associated state-control system.

In this paper we complete the bifurcation analysis of the shallow lake model. We let system parameters vary and we study the dependence of the solution structure on these parameters. We obtain as results two types of planar cuts of the parameter space: first, we keep the biological properties $b$ of a lake fixed and let the socio-economic parameters $c$ and $\rho$ vary; second, we fix the discount rate $\rho$ and let $b$ and $c$ vary. This gives us a fairly complete picture of how the optimal pollution policy for an eco-system responds to changes in the degree of its resilience, social preferences and economic factors.

With the performed analysis we quantify the trade-off between the relative cost of pollution $c$ and the time discount factor $\rho$. In particular for several values of $b$, we compute two-parameter bifurcation diagrams with respect to $c$ and $\rho$ that show that if $\rho$ is decreased, the minimal preference for the environment $c$ that implies that the oligotrophic solution is optimal is decreased proportionally; this solution is characterized by high quality of the lake water and low level of agricultural activity in the long run regardless of the initial pollution level. Thus the oligotrophic solution can be globally optimal in a less environmentally friendly society, if the social planner is sufficiently foresighted.

The main methodological contribution of this paper is systematic use of a proper bifurcation analysis to study non-convex optimal control problems with multiple equilibria. The bifurcation methodology presented in this paper allows one to describe fully the solution structure of a parameterized optimal control problem with multiple equilibria. It enables one to find all possible types of the optimal solutions of such problems, all bifurcations occurring due to varying the parameters. Moreover, bifurcation analysis enables us to in fact analyze an infinity of optimal control models, as each point in the parameter space 
corresponds to a particular problem. A bifurcation diagram gives for each of these particular problems the structure of the set of optimal solutions.

The main ideas of the bifurcation methodology are general and can be applied to a wide range of problems. For example, in Wagener (2005) a problem of optimal investment for firms with non-concave production function and quadratic costs is studied. With the help of bifurcation analysis regions of the cost function's coefficients values are found for which there are multiple local optima of the firm's capital stock and the investment strategy is history-dependent. In Caulkins et al (2007) a model of organizational bridge building is considered, where multiple threshold points are found. In Steindl and Feichtinger (2004) a more complicated problem with a four dimensional state-control space is considered, a production-inventory model with an S-shaped production function. In this model the Hamilton-Hopf bifurcation occurring for zero discounting leads to a family of periodic solutions. With a small perturbation of the system optimal oscillating production processes are obtained.

More generally, the theory of bifurcations of optimal vector fields is potentially relevant to any parameterized optimization problem that does not satisfy the Arrow-Mangasarian sufficiency conditions. For such problems may exhibit indifference threshold points ${ }^{1}$ and associated history-dependent optimal policies.

The outline of the paper is as follows. Section 2 describes the shallow lake model. Section 3 introduces the concept of the optimal vector fields and gives examples of optimal vector fields in the shallow lake model. Section 4 describes different bifurcations of the optimal vector fields and their connection to the bifurcations of a state-control system. Section 5 presents a fairly complete bifurcation analysis of the shallow lake system with respect to all three parameters: the natural rate of pollutant outflow $b$, the relative costs of pollution $c$ and the discount rate $\rho$. Finally, Section 6 concludes.

\section{The Shallow Lake model}

The shallow lake problem is an optimal pollution management problem solved by a social planner. This social planner maximizes a social utility functional, which models conflicting interests of two types of lake users: farmers and "water users", such as tourists, water companies and fishermen. Farmers get benefits from using fertilizers that contain phosphorus; the phosphorus runs off the fields and is eventually washed into the lake. Surplus of phosphorus in water causes growth of aquatic plants that fill the entire water column or that concentrate much of their biomass in the upper water layer. When these plants become dominant the bottom vegetation, which stabilizes the sediment, collapses due to light limitation. As a result surface waves can stir up the sediment - the lake is shallow - and the lake becomes turbid. The drop of water quality leads to losses for the lake users.

The shallow lake model consists of two parts: the pollution dynamics and the social welfare functional. We start with describing the dynamics. Let $x(t)$

\footnotetext{
${ }^{1}$ Also called Skiba points.
} 
be proportional to the amount of phosphorus in the lake at time $t$. The value of $x(t)$ may change due to the input of more phosphorus due to farming activities, $u(t)$, as well as due to sedimentation and the internal biological processes of production of phosphorus. Mäler et al proposed in Mäler et al (2003) to model the pollution dynamics of a shallow lake as

$$
\dot{x}=u-b x+\frac{x^{2}}{1+x^{2}}, x(0)=x_{0},
$$

where $b \geq 0$ is the coefficient that is proportional to the rate of loss of phosphorus due to sedimentation, and where the last term models the biological production process. For more detailed analysis and the biological background of equation (1) see Scheffer (1998).

The second part of the shallow lake model is the social welfare functional. Society in this model consists of lakes users of two types: farmers, who benefit from polluting the lake, and water users, who bear losses when the pollution level increases. The farmers' benefits, or the farmers' utility, is assumed to be an increasing concave function of $u$, taken here $\operatorname{as}^{2} \log u$. The costs of pollution, or the disutility of the water users, is assumed to increase quadratically with the pollution level. Thus the social welfare at time $t$ is $\log u(t)-c x^{2}(t)$, where $c$ is a nonnegative parameter which models the relative cost of pollution.

The total welfare is given by

$$
B[u]=\int_{0}^{\infty}\left(\log u-c x^{2}\right) e^{-\rho t} d t
$$

where $\rho$ is a discount factor and $x$ is defined by (1). The optimal control problem of the social planner is to find the dumping control $u^{*}$ that maximizes the social welfare functional $B$ given the initial level of pollution $x_{0}$ and the pollution dynamics (1). A solution to this optimization problem is a pair $(\tilde{x}, \tilde{u})$, such that $\tilde{u}$ is an admissible control, by which we mean $\tilde{u}$ is piecewise continuous and $\tilde{u}(t) \geq 0 \forall t \geq 0$, and $\tilde{x}$ continuous and piecewise continuously differentiable, (1) is satisfied and $B(\tilde{u}) \geq B(u)$ if $(x, u)$ satisfies (1).

The standard way of solving such a problem is to introduce the current value Hamiltonian

$$
H(x, p, u)=\log u-c x^{2}+p\left(u-b x+\frac{x^{2}}{1+x^{2}}\right),
$$

which has to be maximized with respect to the control variable $u \in \mathbb{R}_{+}$. The additional variable $p \in \mathbb{R}$ is called the co-state and represents the shadow costs of pollution. According to Pontryagin's Maximum Principle, if $u:[0, \infty) \rightarrow(0, \infty)$ is an optimal solution, then $p(t), x(t)$ and $u(t)$ satisfy three conditions:

1) $u(t)$ maximizes the function $h(u)=H(x(t), p(t), u)$ for each $t$. In the shallow lake model this implies the following one-to-one correspondence

\footnotetext{
${ }^{2}$ Results that we obtain in this paper are expected to be fairly robust to the choice of this utility function.
} 
between the costate $p$ and the control $u$

$$
u=U(p)=-\frac{1}{p}
$$

defining the so-called maximized Hamiltonian

$$
\mathcal{H}(x, p)=H(x, p, U(p))
$$

2) $x(t)$ and $p(t)$ are solutions of the reduced canonical system

$$
\left\{\begin{array}{l}
\dot{x}=\frac{\partial \mathcal{H}}{\partial p}(x, p) \\
\dot{p}=\rho p-\frac{\partial \mathcal{H}}{\partial x}(x, p)
\end{array}\right.
$$

3) the transversality condition

$$
\lim _{t \rightarrow \infty} e^{-\rho t} p(t)=0 \quad \text { if } \quad \liminf _{t \rightarrow \infty} x(t)>0 .
$$

is satisfied ${ }^{3}$.

Using (5) and (4) the system (6) reads as

$$
\left\{\begin{array}{l}
\dot{x}=-\frac{1}{p}-b x+\frac{x^{2}}{1+x^{2}} \\
\dot{p}=2 c x+p\left(\rho+b-\frac{2 x}{\left(1+x^{2}\right)^{2}}\right) .
\end{array}\right.
$$

The system (8) is called the shallow lake system. Due to the one-to-one correspondence (4) between the costate $p$ and the control $u$ the shallow lake system (8) can be rewritten in the state-control form

$$
\left\{\begin{array}{l}
\dot{x}=u-b x+\frac{x^{2}}{x^{2}+1} \\
\dot{u}=-\left(\rho+b-\frac{2 x}{\left(x^{2}+1\right)^{2}}\right) u+2 c x u^{2} .
\end{array}\right.
$$

The shallow lake system (9) is a system of parameterized differential equations. Typically in such systems changing the value of the parameters may cause qualitative changes of the solution structure: equilibria may lose stability, new equilibria or attracting sets may appear, etc. Such qualitative changes of the solution structure due to smooth variations of the parameters are called bifurcations. Some bifurcations of the dynamical system (9) affect the optimal pollution policy and consequently the long run pollution level $x$ under the optimal policy. Therefore knowledge of bifurcation values of the system parameters

\footnotetext{
${ }^{3}$ For trajectories such that $\lim _{t \rightarrow \infty} x(t)=0$ the transversality condition is given by $\lim _{t \rightarrow \infty} e^{-\rho t} p(t) \geq 0$.
} 
can shed light upon the long run behavior of the system (9) when the optimal policy is applied.

In the next section we recall the notion of optimal vector fields, which describe the state dynamics under the optimal policy. Also we study correspondence between bifurcations of the optimal vector fields and bifurcations of the dynamical system (9).

\section{Optimal vector fields in the shallow lake model}

This section shortly presents, in an improved form, the results of the bifurcation analysis of the shallow lake system obtained in Wagener (2003) and combines them with the concept of optimal vector fields.

Solutions to the problem of maximizing (2) subject to (1) for fixed $x(0)=x_{0}$ can be represented as a set of initial costates $\mathcal{P}\left(x_{0}\right) \subset \mathbb{R}$ such that if $p_{0} \in \mathcal{P}\left(x_{0}\right)$ then $(x(0), p(0))=\left(x_{0}, p_{0}\right)$ is an initial condition to an optimal trajectory $(x(t), p(t))$ in the state-costate space. Then pair $(x(t), u(t))=(x(t), U(p(t)))$ solves the optimal control problem of the social planner. The set-valued function $\mathcal{P}(x)$ is called the optimal costate rule. The corresponding set-valued function $\mathcal{U}(x)=\{U(p): p \in \mathcal{P}(x)\}$ is called the optimal policy rule.

For problems with one-dimensional state spaces and infinite time horizons the points $\left(x_{0}, p_{0}\right)$ with $p_{0} \in \mathcal{P}\left(x_{0}\right)$ are usually situated on the stable manifolds of a steady state of the state-costate system. If $\mathcal{P}\left(x_{0}\right)$ contains more that one element, then the state is an indifference state. It follows from the principle of optimality that $\mathcal{P}(x(t))$ is single-valued for all $t>0$.

Definition 3.1. The multivalued vector field

$$
\left.X^{O p t}(x)=\frac{\partial \mathcal{H}}{\partial p}(x, \mathcal{P}(x))\right)
$$

is called the optimal vector field. ${ }^{4}$

The notion of optimal vector field is general and not restricted to the shallow lake problem, see Kiseleva and Wagener (in prep.). The optimal vector field determines the direction and the speed of the state flow under the optimal policy. Optimal state trajectories are solutions of

$$
\dot{x}(t)=X^{O p t}(x(t)), \quad x(0)=x_{0} .
$$

Remember though that the optimal policy and consequently the optimal vector field depend upon the system parameters. Hence they may bifurcate when the parameters are varied.

The main result of Wagener (2003) is the bifurcation diagram of the shallow lake system with respect to the parameters $b$ and $c$ for the fixed value of the discount rate $\rho=0.03$. However this bifurcation diagram is incomplete, as

\footnotetext{
${ }^{4}$ Boltyanski in Boltyanski (1966) names these vector fields "synthesized".
} 
there are bifurcation curves missing. In this paper we present the complete version of it in Figure 1(a), which shows the parameter plane $(b, c)$ divided into four regions, labeled respectively: Unique equilibrium, Oligotrophic, Region of history dependence and Eutrophic. Those regions correspond to four different types of solutions of the shallow lake problem, differring in number of equilibria of the state-control system (9) as well as the long run pollution level under the optimal policy. In Figures 1(b)-1(h) we give phase portraits of the statecontrol system (9) for different values of the parameters. Optimal trajectories are represented by thick curves, other trajectories by solid curves. In the upper parts of the phase diagrams the phase plots of the optimal vector fields are given. Attractors of the optimal vector fields are represented by bullets, indifference thresholds by black squares. Later, in Figures 2-6, we denote repellers of the optimal vector fields as circles. We keep these notations throughout the paper.

\section{Unique equilibrium}

For the values of the parameters $b$ and $c$ in this region the state-control system (9) has a unique equilibrium. It is a saddle, see Figure 1(b) and 1(h). The graph of the optimal solution is always situated on the stable manifold of this saddle ${ }^{5}$. The long run pollution level depends then on the values of the parameters $c$ and $b$, changing within the region.

\section{Multiple equilibria}

In cases with multiple equilibria of the state-control system (9) there are always two saddles, denoted as $P=\left(x_{P}, u_{P}\right)$ and $Q=\left(x_{Q}, u_{Q}\right)$. The steady state pollution level $x_{P}$ in $P$ is significantly lower than the pollution level $x_{Q}$ in $Q$; they are called oligotrophic and eutrophic steady states of the lake, respectively. The oligotrophic steady state corresponds to a high level of water services and a low level of agricultural activities, whereas the eutrophic steady state corresponds to a high level of agricultural activities and a low level of water services.

It has been proved in Wagener (2003) that the optimal solution of the social planner optimization problem is situated on the stable manifold of one of the saddles. In the case with multiple equilibria of (9) the social planner has to choose whether to "jump" to the stable manifold of the oligotrophic equilibrium $P$ or to the stable manifold of the eutrophic equilibrium $Q$. Regarding to the choice of the social planner the following three cases are possible:

- the oligotrophic steady state is globally optimal; independently of the initial pollution level of the lake the social planner steers the lake to the clean equilibrium $P$;

- the eutrophic steady state is globally optimal; independently of the initial pollution level of the lake the social planner steers the lake to the turbid equilibrium $Q$;

\footnotetext{
${ }^{5}$ For the proof see Wagener (2003).
} 
- the oligotrophic steady state and the eutrophic steady state are locally optimal; the long run pollution level depends on the initial level of pollution.

Oligotrophic region In the oligotrophic case the optimal trajectory is the stable manifold of the saddle $P$, and the optimal policy is a smooth continuous function of the state, see Figure 1(c). The optimal policy steers the lake to the clean equilibrium $P$ independently of the initial level of the pollution; the clean steady state is globally optimal. The one-dimensional phase diagram of the optimal vector field is drawn in the upper part of the Figure 1(c); it has one attractor, denoted by a bullet.

Eutrophic region In the eutrophic case, see Figure 1(g), the optimal trajectory is the stable manifold of the saddle $Q$. Regardless of the initial level of pollution, the optimal policy steers the lake to the turbid equilibrium. The optimal vector field, drawn in the upper part of the Figure 1(g), has a unique attractor with the whole state space as a basin of attraction; the turbid steady state is globally optimal.

Note that in both cases, oligotrophic and eutrophic, the optimal vector field is single-valued for all initial states.

Region of history dependence History-dependent solutions are distinguished from the other ones by the presence of threshold values of the initial pollution level: if the initial pollution level is below that threshold level then the oligotrophic steady state is optimal, whereas if the initial pollution level is above that threshold level then the eutrophic steady state is optimal. The type of history-dependent solution is determined by the type of the threshold point which can be either a repeller or an indifference point ${ }^{6}$.

Indifference points are initial states $x=x_{0}^{*}$ for which the social planner is indifferent between steering the lake to the clean or to the turbid state; for these states there exist two optimal controls $u_{1}^{*}$ and $u_{2}^{*}$, both maximizing the social welfare functional (2). In the case when the threshold is an indifference point the optimal policy is a smooth single-valued function everywhere, except from a point where it takes two values. That point is the indifference point. The optimal vector field is also multivalued at that point. The indifference point in Figure 1(e) is marked by a black square.

In the case when the threshold is a repeller the optimal policy is a smooth function; it as well as the optimal vector field is everywhere single-valued. This case is not showed in Figure 1, it will be illustrated in the next section.

Threshold points separate two basins of attraction of the optimal dynamics: the states below that point constitute the basin of attraction of the clean equilibrium, and the states above that point constitute the basin of attraction of the turbid equilibrium. Note that the indifference point lies in both basins,

\footnotetext{
${ }^{6}$ Indifference points are also called Skiba points, DNS points or DNSS points. For the naming see Grass et al (2008) p.238.
} 


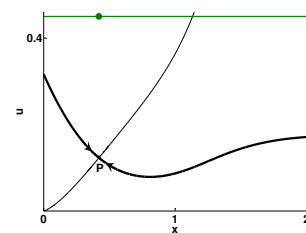

(b) Unique equilibrium, $\quad c=0.7$

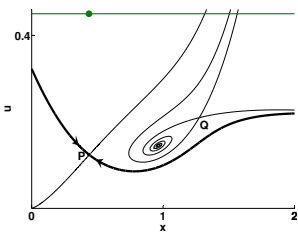

(c) Oligotrophic solution, $\quad c=0.55$

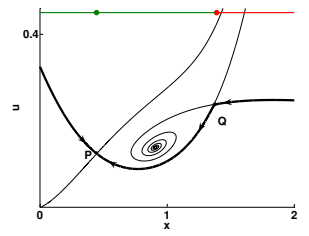

(d) Lower heteroclinic connection, $c=0.53523$
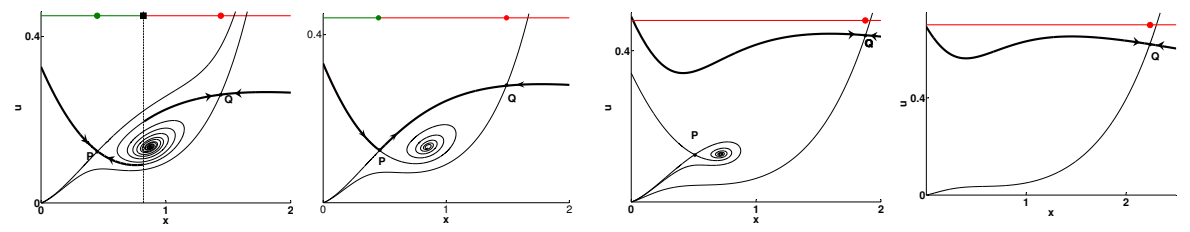

(e) History-dependent (f) Upper hetero- (g) Eutrophic solu- (h) Unique equilibsolution, $c=0.5$ clinic connection, tion, $c=0.47379$

Figure 1: Figure 1(a) shows the bifurcation diagram of the shallow lake system in the $(b, c)$-parameter space for $\rho=0.03$. Dashed lines represent saddle-node bifurcation curves, separating the region of parameters for which there is a unique equilibrium in the system from the region of multiple equilibria. Solid lines indicate heteroclinic bifurcation curves. Phase portraits of state-control system and of the optimal vector fields are given for $b=0.65$ and selected values of $c$. Optimal trajectories are represented by thick curves, other trajectories are represented by solid curves. Optimal solutions are always situated on the stable manifold of one of the saddles. In the upper parts of the phase diagrams the phase plots of the optimal vector fields are drawn. Attractors of the optimal vector fields are denoted by bullets, indifference points by squares. 
whereas the repeller lies in neither of them. The history-dependent pollution policy steers the lake to the clean equilibrium only if the lake is initially not very polluted, otherwise it steers the lake to the turbid equilibrium.

One can see from Figure 1 that in all cases except the history-dependent case the optimal vector field has only one attractor, whereas in the historydependence case it has two attractors and one threshold point. Those optimal vector fields correspond to different values of the parameter $c$, hence as the parameter $c$ varies the optimal vector field undergoes a bifurcation. Since the optimal vector fields depend upon the optimal policies, bifurcations of the optimal vector fields are connected with bifurcations of the state-control system.

In the next section the classification of bifurcations of optimal vector fields is shortly recalled from Kiseleva and Wagener (in prep.) and their connections to the bifurcations of the state-control space are studied.

\section{Bifurcations of optimal vector fields}

In this section we describe the codim 1 and codim 2 bifurcations of one-dimensional optimal vector fields that occur in the shallow lake problem. Recall that the codimension of a bifurcation is the number of parameters which must be varied for the bifurcation to occur. This corresponds to the codimension of the parameter set for which the bifurcation occurs within the full space of parameters. We shall illustrate these bifurcations by referring to the relevant parts of Figure 1.

\subsection{Indifference-attractor bifurcation}

First we describe the indifference-attractor (IA) bifurcation. When the optimal vector field passing through this bifurcation, an attractor and an indifference point of the optimal vector field are created. In the state-control space this corresponds to the unstable manifold of one of the saddles and the stable manifold of the other saddle changing their relative positions, going through a heteroclinic bifurcation.

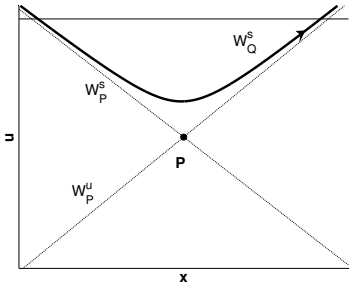

(a) Before bifurcation

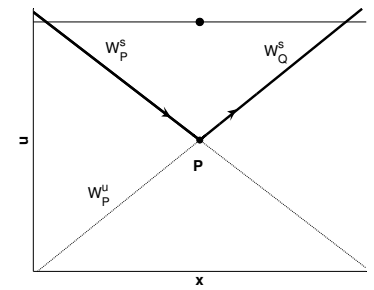

(b) At bifurcation

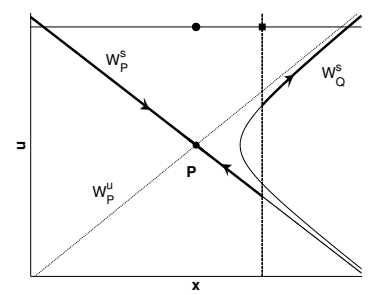

(c) After bifurcation

Figure 2: The indifference-attractor bifurcation. Legend as in Figure 1. 
The situation is illustrated in Figures 1(g)-1(f)-1(e). Blow-ups in the vicinity of the left saddle $P$ are shown in Figure 2. Before the bifurcation, the stable manifold $W_{Q}^{s}$ of the right saddle $Q$ "covers" $P$, meaning that there exists $w$ such that $\left(x_{P}, w\right) \in W_{Q}^{s}$. Hence $W_{Q}^{s}$ is the optimal trajectory, see Figure 2(a). This is the same situation as in Figure $1(\mathrm{~g})$. At the bifurcation the left part of the stable manifold $W_{Q}^{s}$ coincides with the right part of the unstable manifold $W_{P}^{u}$; the manifolds form what is called a heteroclinic connection of the two saddles, see Figure 2(b) and Figure 1(f). After the bifurcation, the trajectory $P$ is optimal, and all optimal state trajectories starting in a neighborhood around $x_{P}$ converge towards $x_{P}$, see Figure 2(c) and compare to Figure 1(e). The length of these neighborhoods is bounded on the right by an indifference point, from which the system can optimally go to either $x_{P}$ or $x_{Q}$.

\section{$4.2 \quad$ Indifference-repeller bifurcations}

The second bifurcation to be described is the indifference-repeller bifurcation. This bifurcation is not illustrated in Figure 1; the indifference-repeller bifurcation curves are not visible there, since they are located in the very corner between the two dashed curves. They become visible in blow up, that is shown in Figure 14.

There are two types of indifference-repeller bifurcations. In both cases an indifference point of the optimal vector field changes into an unstable steady state; however the corresponding changes of the state-control space follow different scenarios.

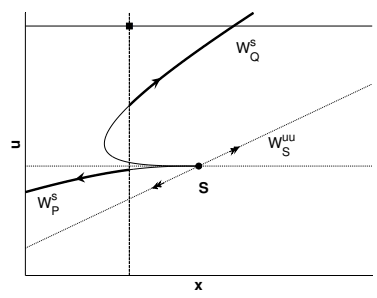

(a) Before bifurcation

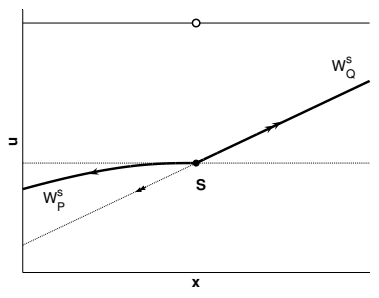

(b) At bifurcation

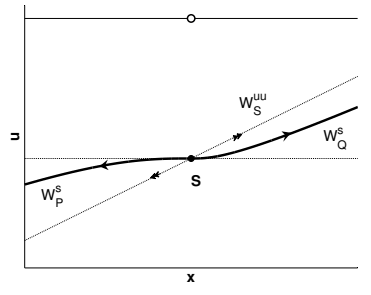

(c) After bifurcation

Figure 3: The indifference-repeller bifurcation of type one. Legend as in Figure 1.

Figure 3 displays the scenario of an indifference-repeller bifurcation of type one, denoted as "IR1", in a neighborhood of the central repelling steady state $S=\left(x_{S}, u_{S}\right)$ of the state-control system. This state has two positive eigenvalues $0<\lambda^{u}<\lambda^{u u}$. To the largest eigenvalue, a unique one-dimensional manifold is associated, the so-called strong unstable manifold $W_{S}^{u u}$ : this is the trajectory that approaches $S$ at the rate $e^{\lambda^{u u} t}$ as $t \rightarrow-\infty$. The strong unstable manifold $W_{S}^{u u}$ is represented in Figure 3 by the curve with two arrows. At the bifurcation, 
the relative positions of the strong unstable manifold $W_{S}^{u u}$ and the stable manifold $W_{Q}^{s}$ change. Before the bifurcation, Figure 4.2, the manifold $W_{Q}^{s}$ "covers" $S$, which is then not optimal. But $W_{Q}^{s}$ does not cover all of the state space and hence there is an indifference point close to $x_{S}$. After the bifurcation the steady state $S$, though repelling, is optimal. The corresponding state $x_{S}$, while not an indifference point any more, is still a threshold point: in every neighborhood of $x_{S}$ there are optimal trajectories tending to different long-term steady states.

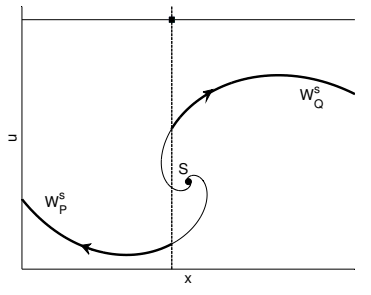

(a) Before bifurcation

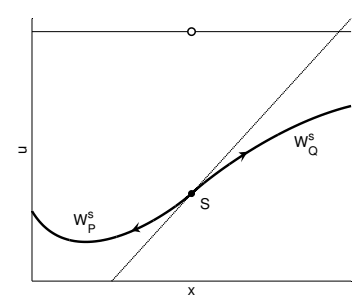

(b) At bifurcation

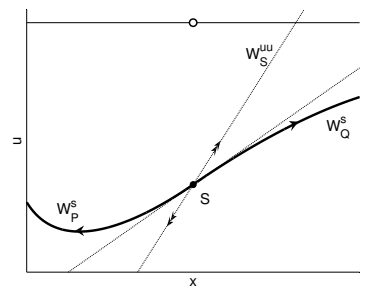

(c) After bifurcation

Figure 4: The indifference-repeller bifurcation of type two. Legend as in Figure 1.

Figure 4 displays the scenario of an indifference-repeller bifurcation of type two, denoted as "IR2", in a neighborhood of the central repelling steady state $S=\left(x_{S}, u_{S}\right)$ of the state-control system. Before the bifurcation the steady state $S$ has two complex eigenvalues $\lambda_{1,2}=\mu \pm i \alpha$ with positive real parts $\mu>0$. The stable manifolds $W_{Q}^{s}$ and $W_{P}^{s}$ of the saddles $Q$ and $P$ are spiraling from the source $S$, giving rise to an indifference point close to $x_{S}$, see Figure 4(a). At the bifurcation the eigenvalues of the steady state $S$ become equal and real. The steady state itself becomes a degenerate node and all trajectories move away from $S$ in the direction of a unique eigenvector, see Figure 4(b). After the bifurcation the steady state $S$ has two real positive eigenvalues $0<\lambda^{u}<\lambda^{u u}$ and the unique eigenvector splits up into two eigenvectors $e^{u}$ and $e^{u u}$. Almost all the trajectories move away from $S$ in the direction of the eigenvector $e^{u}$; again the steady state $S$ itself, though repelling, is an optimal trajectory, see Figure 4(c).

\subsection{Other bifurcations}

Optimal vector fields can also undergo "standard" saddle-node "SN" and cusp "C" bifurcations, which are associated to saddle-node and cusp bifurcations of the state-control system. However, not all bifurcations of the state-control system imply bifurcations of the optimal vector field. As an example consider the saddle-node bifurcation of the state-control system illustrated in Figures 1(b)-1(c). Though two new equilibria appear, the structure of the optimal control strategies does not change, and the stable manifold of the saddle $P$ remains the 


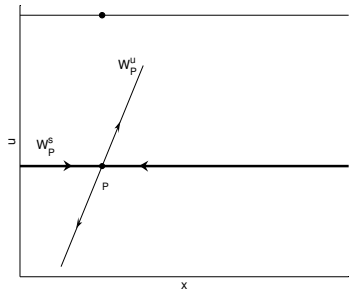

(a) Before bifurcation

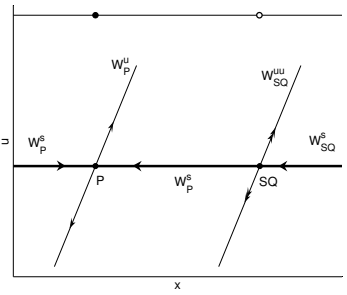

(b) At bifurcation

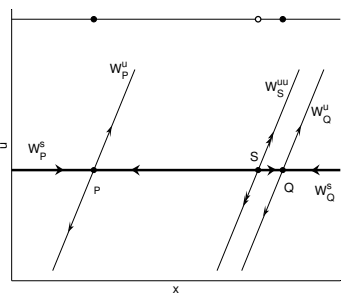

(c) After bifurcation

Figure 5: The saddle-node bifurcation of the optimal vector field. Legend as in Figure 1.

optimal trajectory. Consequently, the optimal vector field does not bifurcate. We therefore call this bifurcation of the state-control system inessential to the optimal vector field. A bifurcation of the state-control system that changes the optimal vector field will be called essential.

The same scenario is represented in Figures $1(\mathrm{~g})$ and $1(\mathrm{~h})$, but with the stable manifold of the saddle $Q$ as the optimal trajectory. An example of a saddle-node bifurcation of the state-control system implying saddle-node bifurcation of the optimal vector field is depicted in Figure 5. The state-control system undergoes a standard saddle-node bifurcation: as a parameter changes a saddle and an unstable node are created. After the bifurcation the stable manifolds of two saddles are connected to the central repelling steady state. The optimal trajectories are then the two stable manifolds and all three equilibria. In particular, the repelling equilibrium of the state-control system corresponds to a repeller of the optimal vector field.
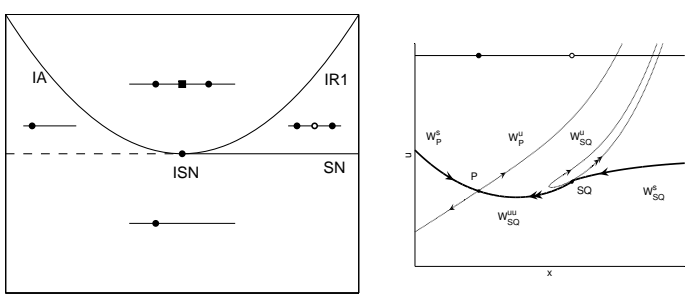

Figure 6: The indifference-saddle-node bifurcation: the phase plot at bifurcation (right) and bifurcation curves emerging from the "ISN" point (left). Legend as in Figure 1.

More complicatedly, we can have the situation that at a saddle-node bifurcation of the state-control system the strongly unstable manifold of the bifurcating steady state coincides with the stable manifold of the non-bifurcating 
saddle. This gives rise to what we call in Kiseleva and Wagener (in prep.) an indifference-saddle-node bifurcation "ISN"; it is a codimension two bifurcation, typically occurring only if there are two or more system parameters. From such a point indifference-attractor, indifference-repeller, saddle-node and inessential saddle-node curves emanate, see Figure 6.

Another codimension two bifurcation is the double-indifference-repeller bifurcation "DIR", when two conditions are satisfied: the repeller $S$ is a degenerate node and the stable manifold of one of the saddles $P$ or $Q$ is connected to the unique eigenvector of $S$. The optimal trajectories are both stable manifolds of the saddles $P$ and $Q$ and the repeller $S$. The optimal vector field has two attractors separated by a repeller. This situation is drawn in Figure 7, where the stable manifold of $Q$ is connected to the unstable manifold of $S$.
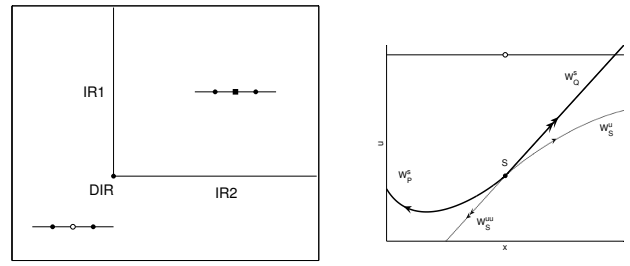

Figure 7: The double-indifference-repeller bifurcation: the phase plot at bifurcation (right) and bifurcation curves emerging from the "ISN" point (left). Legend as in Figure 1.

\section{Bifurcating optimal vector fields of the shal- low lake system}

This section studies the dependence of the optimal vector fields upon the system parameters: the natural rate of sedimentation $b$, the relative weight of ecological services $c$ and the discount rate $\rho$. We apply the bifurcation analysis described in the previous section to compute two-parameter bifurcation diagrams. Such a diagram is a partition of the parameter plane into $2 \mathrm{D}$ regions of structural stability of a dynamical system, 1D bifurcation curves and bifurcation points. Different regions of the parameter-plane correspond to qualitatively different types of the solution structure of the dynamical system. In the shallow lake system the solution structure defines the type of the optimal solution and therefore the type of the optimal vector field. Hence different regions of bifurcation diagrams correspond to structurally different optimal solutions and structurally different optimal vector fields. Moreover we distinguish between bifurcation curves of the shallow lake system itself and bifurcation curves of the optimal vector fields. 


\subsection{No discounting case}

We begin with considering the shallow lake model without discounting, i.e. $\rho=0$ in (6). In this case, we use overtaking optimality ${ }^{7}$ as our optimality criterion. Recall that an admissible control $u^{*}$ is called overtaking optimal if, for any admissible control $u$, there is a $T(u)$ such that for every $T \geq T(u)$

$$
B_{T}(u) \leq B_{T}\left(u^{*}\right)
$$

where

$$
B_{T}(u)=\int_{0}^{T}\left(\log u-c x^{2}\right) e^{-\rho t} d t .
$$

and where $x$ satisfies (1).

For $\rho=0$ the shallow lake system becomes Hamiltonian. Then trajectories of (8) are level curves of the maximized Hamiltonian $\mathcal{H}(x, p)$ given in (5) and steady states are critical points of $\mathcal{H}(x, p)$. Due to the one-to-one state-costate correspondence (4) the level curves of $\mathcal{H}(x, p)$ correspond to the level curves of $\mathcal{H}(x,-1 / u)$ in the state-control space $(x, u)$.

For solutions on the stable manifolds of $P$ or $Q$ we have

$$
B_{T}(u)=\mathcal{H}(P) T+o(T) \text { as } T \rightarrow \infty
$$

and

$$
B_{T}(u)=\mathcal{H}(Q) T+o(T) \text { as } T \rightarrow \infty .
$$

Since optimal solutions converge to either $P$ or $Q$ overtaking optimality is determined by the values $\mathcal{H}(P)=\log u_{P}-c x_{P}^{2}$ and $\mathcal{H}(Q)=\log u_{Q}-c x_{Q}^{2}$. More precisely, the trajectory converging to $P$ will be preferable in the sense of overtaking optimality if $\mathcal{H}(P)>\mathcal{H}(Q)$ and vice versa.

Figure 8 shows the bifurcation diagram of the shallow lake system (9) for $\rho=0$. Due to the fact that the system is Hamiltonian there is only one curve of heteroclinic bifurcations, which ends at the cusp point "C". The vertical branch of the indifference-attractor bifurcation curve "IA" is located on the straight line $b=1 / 2$ and separates two regions: the oligotrophic region and the region of history-dependent optimal policies. However the corresponding bifurcation scenario in the state-control space is different from the one described in Section 4.

Let us compare phase plots of the shallow lake system (9) for $b=0.6$ and $b=0.4$ while other parameters are held fixed $\rho=0, c=0.5$.

First we fix $b=0.6$. In this case the shallow lake system has three equilibria with non-zero control: two saddles and one center, see Figure 9(left). Since the only candidates for the optimal solution are the stable manifolds of the two saddles $P$ and $Q$, we have to compare the values $\mathcal{H}(P)$ and $\mathcal{H}(Q)$ and choose the largest one. Note that the stable manifold of $P$ covers the whole state space, therefore it can be presented as a smooth continuous function $u=w_{P}^{s}(x)$ with the property $\mathcal{H}\left(x, w_{P}^{s}(x)\right)=\mathcal{H}(P)$. Since for $(x, u)$ such

\footnotetext{
${ }^{7}$ For a detailed discussion of this criterion see Grass et al (2008).
} 


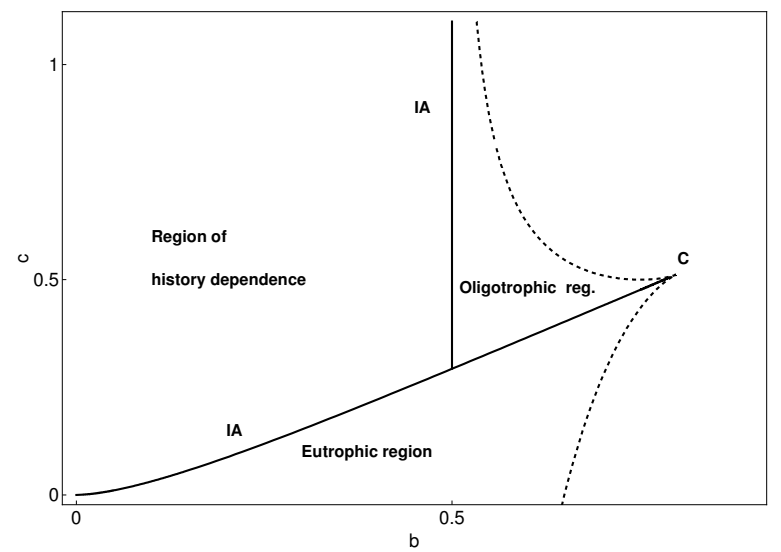

Figure 8: Bifurcation diagram of the shallow lake system for $\rho=0$.

that $u<b x-x^{2} /\left(1+x^{2}\right)$ we have that $\partial \mathcal{H}(x, u) / \partial u<0$, we conclude that $\mathcal{H}(P)=\mathcal{H}\left(x_{Q}, w_{P}^{s}\left(x_{Q}\right)\right)>\mathcal{H}(Q)$ and the stable manifold of $P$ is the optimal trajectory, see Figure 9 (left).
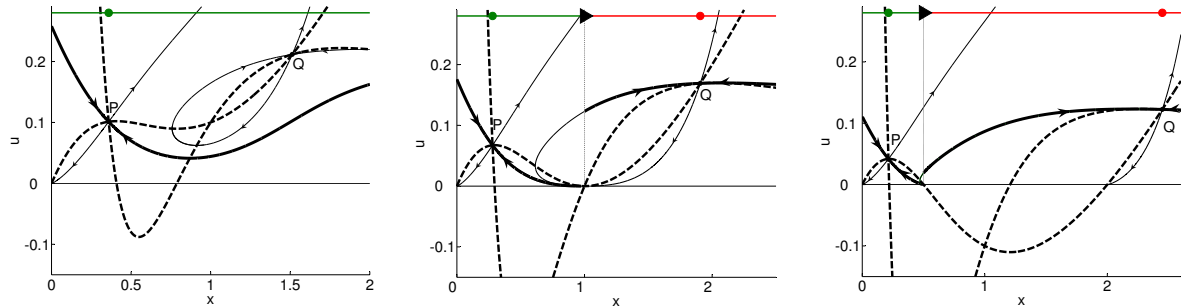

Figure 9: Phase plots of the shallow lake system for $\rho=0, c=0.5, b=0.6$ (left), $b=0.5$ (middle) and $b=0.4$ (right). Solid lines represent invariant manifolds of the two saddles $P$ and $Q$, dashed lines represent the isoclines $\dot{x}=0$ and $\dot{u}=0$. The optimal solution is represented by thick curves.

Now let us fix $b=0.4$. Recall that for $b<1 / 2$ the shallow lake system is irreversible, meaning that the lower heteroclinic connections of the saddles are impossible. For this value of $b$ the shallow lake system has two equilibria with non-zero control: the two saddles $P$ and $Q$. The only candidates for the optimal solution are their stable manifolds. The stable manifold of the oligotrophic saddle $P$ does not cover the whole state space. In fact, it covers the interval $[0, \hat{x}]$, where $\hat{x}=\left(1-\sqrt{1-4 b^{2}}\right) /(2 b)$ is the smallest positive $x$-coordinate of intersection of the isocline $\dot{x}=0$ and the axis $u=0$. If the initial level of pollution $x(0) \geq \hat{x}$ then it is not possible to steer the lake to the oligotrophic 
steady state. In order to find the optimal trajectory let us compare the values
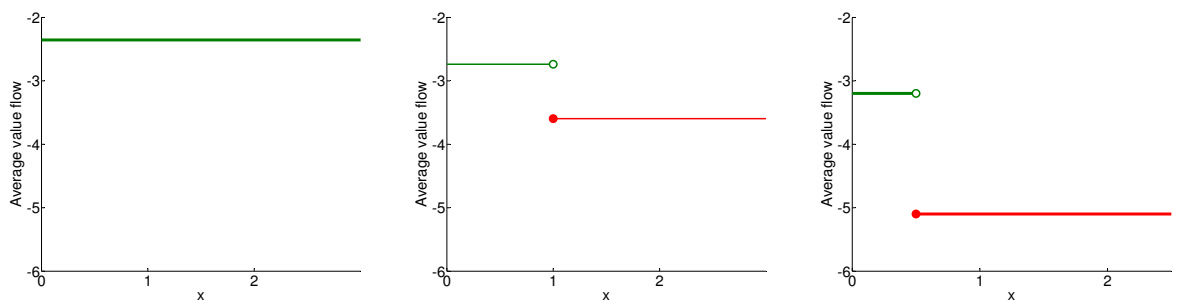

Figure 10: Average value flows corresponding to the optimal solutions of the shallow lake model for $\rho=0, c=0.5$ and $b=0.6$ (left), $b=0.5$ (middle), $b=0.4$ (right). In case of history-dependent optimal policy the value function is discontinuous.

$\mathcal{H}(P)$ and $\mathcal{H}(Q)$. Analogously to the case $b=0.6$ we compare these values for $x$ located in the left neighborhood of $x=\hat{x}$. We conclude that $\mathcal{H}(P)>\mathcal{H}(Q)$ implying that the stable manifold of $P$ is the optimal trajectory. However the corresponding optimal policy is available only for $x<\hat{x}$, and for $x \geq \hat{x}$ the optimal solution lies on the stable manifold of $Q$, see Figure 9(right). The point $x=\hat{x}$ in this case is called an irreversibility threshold. In Figure 9 we denote this point by a black triangle.

It is important to note here that in case of no discounting the value function corresponding to the optimal solution is infinite. Instead we consider the average value flows

$$
v=\lim _{T \rightarrow \infty} \frac{1}{T} B_{T}(u)=\mathcal{H}\left(u_{\infty}\right) .
$$

The average value flows corresponding to the optimal solutions are discontinuous if $\rho=0$ and $(b, c)$ take values in the "Region of history dependence". Figure 10 shows the average value flows for the three types of the optimal solution presented in Figure 9. For $b=0.5$ and $b=0.4$ they are discontinuous at the irreversibility threshold point.

\subsection{Weight of ecological services versus discounting}

Now we fix the value of the decay rate $b$ and we take the relative economic weight of pollution $c$ and the discount rate $\rho$ as bifurcation parameters. This kind of analysis allows us to study the dependence of the optimal pollution policy upon social preferences, while the biological properties of the lake are assumed to be given. The bifurcation diagram, given in Figure 11, displays the results of a "comparative dynamics" analysis of the system, as it indicates how the total solution structure changes with the parameters. All the curves in Figure 11 are bifurcations curves of the shallow lake system, but as we mentioned above some bifurcations of the state-control system are irrelevant to the optimal vector 


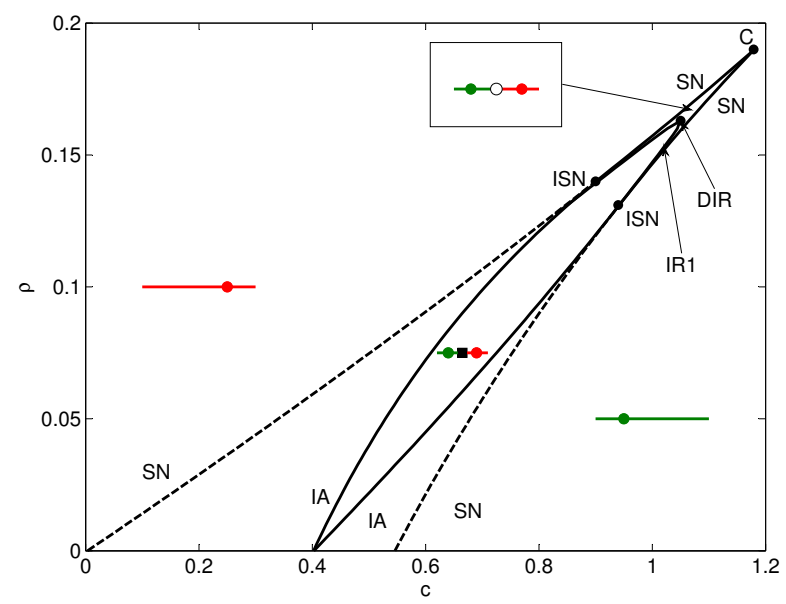

Figure 11: The bifurcation diagram of the shallow lake system in the $(c, \rho)$ parameter space for $b=0.65$. Solid lines represent bifurcation curves of the optimal vector field, dashed lines represent bifurcation curves of the state-control system, that are not bifurcation curves of the optimal vector field.

field. To distinguish such irrelevant bifurcation curves we draw them as dashed curves. The solid bifurcation curves represent curves of essential bifurcations, that is, bifurcations of the optimal vector field. In Figure 11 essential curves divide the parameter space into three separate regions. In the outer region, the optimal vector field $X^{O p t}$ has a unique global attractor. For parameters taking values in the lower inner region, $X^{O p t}$ has two attractors, separated by an indifference point. In the small upper inner region, there are again two attractors but separated by a repeller. All three steady states are engaged in the cusp bifurcation which marks the point with the largest value $\bar{\rho}(b)$ of $\rho$ in the inner region, which is the supremum of values of $\rho$ such that the optimal vector field can have three equilibria.

The union of the two inner regions is the region where there are multiple long-term steady states: we might also call it the region of history-dependence. Consider what happens when we fix $\rho=0.05$ and decrease $c$ from $c=1$ towards $c=0$. If $c$ is large, it is always optimal to steer the lake towards a clean "oligotrophic" long-term steady state. Then at $c \approx 0.61$, we enter the region of history-dependence: if the lake is initially sufficiently clean, it is still optimal to steer it towards a clean state. However, if the lake is initially already too polluted, this is not worthwhile any more. Finally, at $c \approx 0.54$, the basin of attraction of the oligotrophic state collapses, and we enter the region where there is again a single long-term optimal steady state, but now a polluted "eutrophic" one.

Increasing the discount rate $\rho$ has the same effect as decreasing the economic 
weight of the lake $c$. This is according to our intuition, since both increasing the discount rate and decreasing the weight of the stock-damage term in the utility functional decreases the importance attached to long-term effects. For parameters in the region $\rho>\bar{\rho}$, there is always a single, globally attracting steady state, which depends continuously on $c$ and $\rho>\bar{\rho}$.

Effects of varying the natural rate of decay The parameter space can be divided into four regions, according to the values of $b: b \in I_{i}, i=1, \ldots 4$, where $I_{1}=[0,1 / 2], I_{2}=\left(1 / 2, b_{1}^{*}\right], I_{3}=\left(b_{1}^{*}, b_{2}^{*}\right), I_{4}=\left[b_{2}^{*},+\infty\right)$, with

$$
\begin{aligned}
& b_{1}^{*}=\frac{(75-43 \sqrt{3}) \sqrt{-3+2 \sqrt{3}}}{8(-161+93 \sqrt{3})} \approx 0.5505, \\
& b_{2}^{*}=\frac{3 \sqrt{3}}{8} \approx 0.6495 .
\end{aligned}
$$

For $b \in I_{4}$, the regeneration function $g(x)=-b x+x^{2} /\left(1+x^{2}\right)$ in the state dynamics equation $\dot{x}=u-g(x)$ is monotonic. In this case, to every constant loading level $\bar{u}$, satisfying $\bar{u}=g(\bar{x})$ there corresponds a unique pollution level $\bar{x}$, and $\bar{x}$ depends continuously on $\bar{u}$. For $b \in I_{1} \cup I_{2} \cup I_{3}$ the regeneration function is not monotonic, and there catastrophic jumps in the pollution level are possible as the constant level $\bar{u}$ gradually increases, see Mäler et al (2003) and Wagener (2009). However for $b \in I_{2} \cup I_{3}$ catastrophic shifts in the pollution can be reversed by decreasing $\bar{u}$ sufficiently, while for $b \in I_{1}$ they are not reversible as the self-cleaning ability of the lake is insufficient for these values of $b$.

Figure 12 displays the bifurcation diagram of the shallow lake system for $b=0.55$. With a decrease in $b$ the saddle-node bifurcation lines move away from each other, expanding the lower region of history-dependence. We can show that the upper region of history-dependence is unbounded, because there does not exist a cusp point for $b \leq b_{1}^{*}$. For the proof see Appendix A. This implies that the region where the optimal vector field $X^{O p t}$ has a unique global attractor is now separated into two regions. It can be shown that for any nonnegative value $\rho=\rho^{*}$ of the discount rate the pollution level is lower for $\left(c, \rho^{*}\right)$ in the right lower region than for $\left(c, \rho^{*}\right)$ from the left upper region. Note also that for any positive value of the parameter $c$ there exists a value of the discount rate $\rho$ such that the shallow lake system ends up in an equilibrium with a relatively high pollution level.

Finally let us consider the case of an irreversible system, $b \in I_{1}$. In Figure 13 the bifurcation diagram of the shallow lake system for $b=0.45$ is displayed. One can immediately notice that the saddle-node bifurcation curve corresponding to the genesis of the eutrophic equilibrium, that is the right "SN" curve in Figure 12, and the indifference-attractor bifurcation curve corresponding to the lower heteroclinic connection of the saddles, that is the right "IA" curve in Figure 12, are absent in Figure 13. The disappearance of the "SN" curve is explained by the following proposition.

Proposition 5.1. The saddle-node bifurcation curves in the $(b, c)$-plane that 


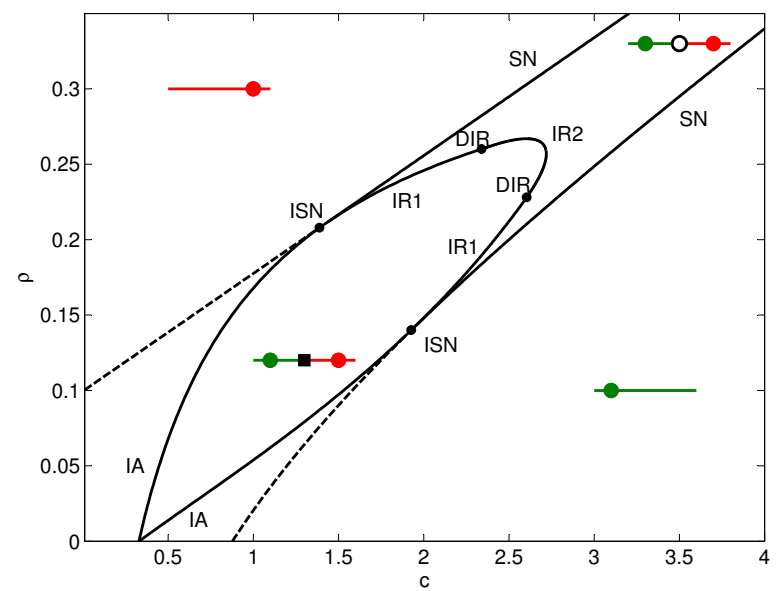

Figure 12: The bifurcation diagram of the shallow lake system in the $(c, \rho)$ parameter space for $b=0.55$. Solid lines represent bifurcation curves of the optimal vector field, dashed lines represent bifurcation curves of the state-control system, that are not bifurcation curves of the optimal vector field.

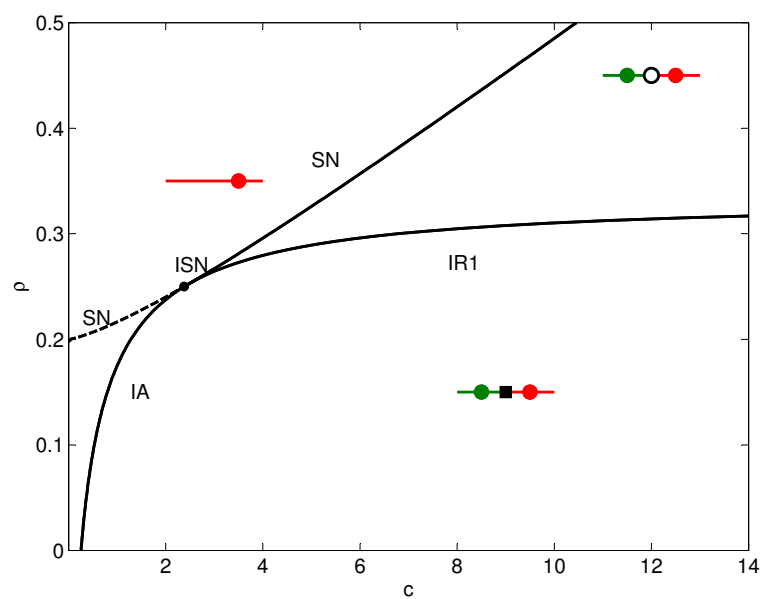

Figure 13: The bifurcation diagram of the shallow lake system in the $(c, \rho)$ parameter space for $b=0.45$. Solid lines represent bifurcation curves of the optimal vector field, dashed lines represent bifurcation curves of the state-control system, that are not bifurcation curves of the optimal vector field. 
correspond to the genesis of the eutrophic equilibrium have a vertical asymptote $b=1 / 2$ for any positive value of $\rho$.

Proof. See Appendix B.

A more detailed discussion of this fact is given in the next subsection. The disappearance of the "IA" curve can be explained by the impossibility of a lower heteroclinic connection of the two saddles. Recall that if the system is reversible it is possible to steer the lake to the clean equilibrium $P$ starting in a neighborhood of the polluted one $Q$, as in Figure 1(d). However in case of irreversibility the stable manifold of $P$ cannot be connected to the unstable manifold of $Q$.

Therefore, for $b<1 / 2$, the eutrophic equilibrium is not involved in any saddle-node bifurcation, meaning that it always exists, nor in any indifferenceattractor bifurcation, meaning that it is always locally optimal. This explains why there is only one saddle-node bifurcation curve and only one indifference attractor bifurcation curve in Figure 13.

\subsection{Weight of ecological services versus natural rate of decay: the discounted case}

Figure 14 shows the bifurcation diagram of the optimal vector field $X^{O p t}$ for $\rho=0.03$ and its blow up near the cusp point "C". The indifference-repeller bifurcation curves "IR1" and "IR2" are situated in the very corner between the saddle-node bifurcation curves "SN". The "IR1" curves are almost coinciding with these in the vicinity of the "ISN" points, see Figure 14(right). As
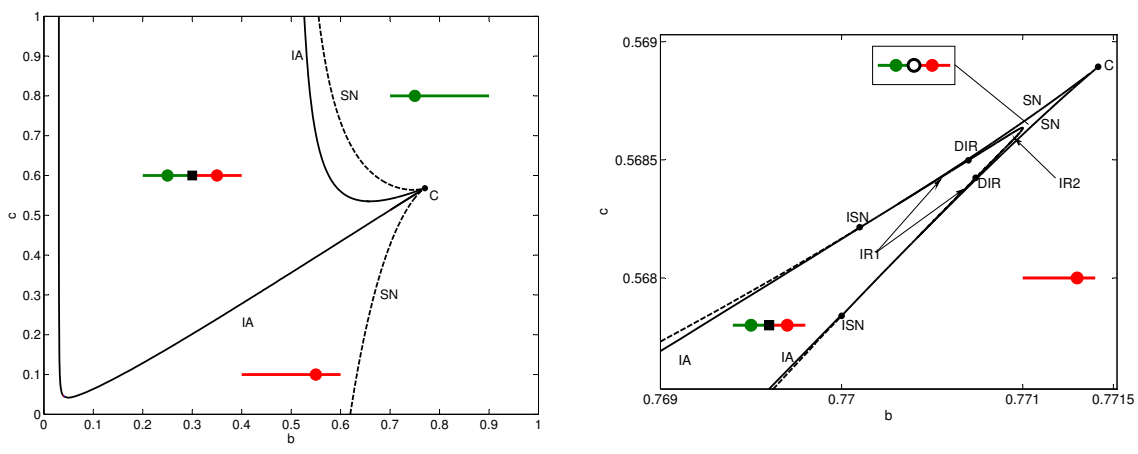

Figure 14: The bifurcation diagram of the optimal vector field $X^{O p t}$ in the $(b, c)$-plain for $\rho=0.03$ (left) and its blow up near the cusp point (right).

$\rho$ increases the "ISN" points move away from the cusp point "C" along the saddle-node bifurcation curves. 
In fact there exist three saddle-node bifurcation curves of the shallow lake system in Figure 14(left): two of them meet at the cusp point "C". The third one exists only for large values of the parameter $c$; therefore we do not see it in Figure 14. However when $\rho$ increases it moves down, as shown in Figure 15, where it appears in the upper left-hand corner.

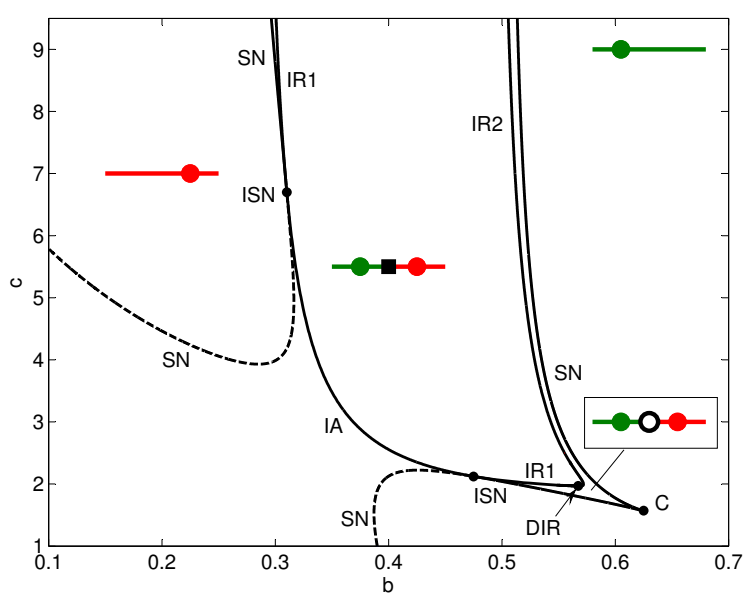

Figure 15: Bifurcation diagram of the shallow lake system with respect to the parameters $b$ and $c$ for $\rho=0.245$.

Figure 15 displays the bifurcation diagram in the $(b, c)$-plane for $\rho=0.245$. For this value of $\rho$ the bifurcation curves divide the parameter plane into three regions that correspond to qualitatively different optimal vector fields. The inner region, which is bounded by two "IR1", one "IR2" and one "IA" curve, corresponds to a history-dependent solution with an indifference point as a threshold. This region, together with two others that are located between "SN" and "IR1", "IR2" curves, and that correspond to a history-dependent solution with a repeller as a threshold, form a region that we call a region of history dependence. The outer region is called the region of uniqueness. In the region of history dependence the optimal vector field $X^{O p t}$ has two attractors separated by either a repeller or an indifference point, implying history dependence of the long run pollution level under the optimal policy. In the region of uniqueness the optimal vector field $X^{O p t}$ has a unique attractor. The bifurcation diagram can be interpreted as follows. Assume that relative cost of pollution $c$ is high, which implies relatively low phosphorus loading. If the natural outflow of the pollutant in a lake is a fast process, i.e. $b$ is high, then the lake is able to sustain low phosphorus loading for any initial level of pollution. But if $b$ is low then the pollutant accumulates in the water, and even an initially clean lake is not able to remain clean in presence of constant phosphorus loading. Now assume that relative cost of pollution $c$ is low, then the initial state of a lake does not affect 
its long run pollution level due to heavy phosphorus loading.

For the critical parameter value $\rho=1 / 4$ the saddle-node bifurcation curves which exist for $b \leq 1 / 2$ meet each other, see Figure 16(left), and for $\rho>1 / 4$
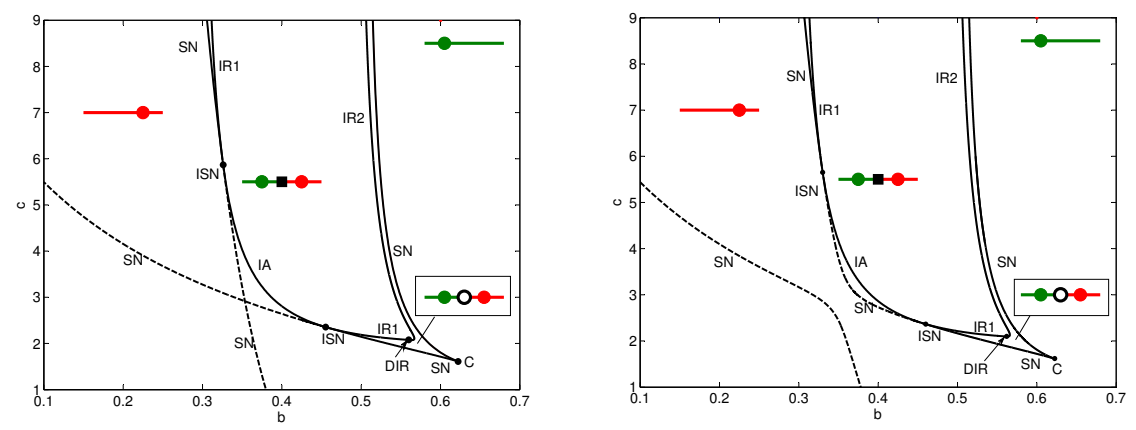

Figure 16: Bifurcation diagram of the shallow lake system in the parameter plane $(b, c)$ for $\rho=0.25$ (left) and for $\rho=0.251$ (right).

they split again but in a different manner, see Figure 16(right), giving rise to a separate saddle-node bifurcation curve, the dashed curve in the low left-hand corner in Figure 16(right). These bifurcations are irrelevant to the optimal vector field, as it is a curve of saddle-node bifurcations of the state-control system (9) which do not cause any bifurcations of the optimal vector field $X^{O p t}$.

The region of history-dependent optimal policies shrinks and moves up as the discount factor $\rho$ increases, see Figure 17. One can see that the right boundary of this region hardly moves, whereas the left boundary moves quickly as $\rho$ increases. This fact can be explained as follows. Assume the natural rate of decay to be small, i.e. $b \leq 1 / 2$, meaning that the lake accumulates most of the pollutant coming in. With an increase in $\rho$ the social planner becomes more myopic. The more myopic optimal policy allows for heavier pollutant loading. Thus, due to the low self-cleaning ability of the lake and the heavy phosphorus loading, the pollution level rapidly converges to a high steady level regardless of its initial value. However, if $c$ is high enough, i.e. the society is sufficiently concerned about the ecosystem quality, the optimal policy becomes history-dependent, and thereby the initial state of the lake determines its long run pollution level. To see this, note that the saddle-node curves that band the region of historydependence on the left have an asymptote $b=0$; this is proved in Appendix B. This implies that for any $0<b \leq 1 / 2$ and $\rho>0$ there is a value of $c$, possible large, such that $(b, c, \rho)$ is in the region of history-dependence.

\section{Concluding remarks}

This paper applies the tools of exploring dynamic optimization problems with multiple equilibria to the shallow lake model. These tools, the notion of optimal 


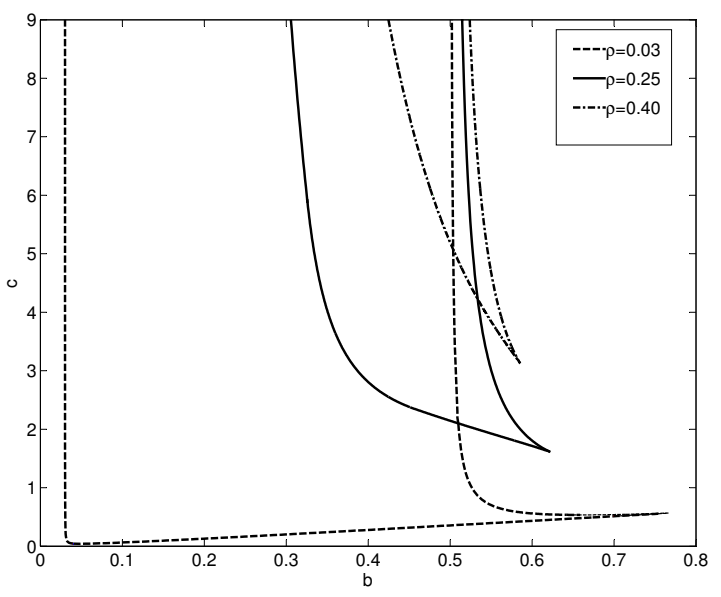

Figure 17: Regions of history-dependent optimal policy for different values of the discount rate $\rho$.

vector fields and the theory of bifurcating optimal vector fields, has been introduced and described in Kiseleva and Wagener (in prep.). The present paper illustrates how they work for a particular optimization problem. These tools however can be applied to a wide class of economic problems.

With the help of the proper bifurcation analysis we have succeeded to complete the analysis of the shallow lake model started in Wagener (2003). We have built the full picture of all possible qualitatively different optimal pollution policies depending on the type of an eco-system, social preferences and economic factors. Moreover we have computed boundaries of the regions in the parameter space that correspond to different types of optimal policies. Each point in the parameter space determines a particular optimization problem. A certain type of the optimal solution corresponds to a point in a particular region, and an intermediate degenerate situation between two types of the optimal solution corresponds to a point on a boundary.

Roughly speaking there are three types of the optimal solution: 1) steering a system to an equilibrium level regardless of its initial state; 2) steering a system to either of two existing equilibria depending on its initial state; 3) steering a system to either of the two equilibria unless the initial state is not at its intermediate steady state level. The last two types of the optimal solution are called history-dependent optimal policies.

The two types of history-dependent optimal policies are distinguished only by the type of the threshold point: it is either an indifference point or a repeller of the optimal vector field. In the shallow lake model, in the first case if the initial pollution level is at the threshold value then the social planner is free to decide which equilibrium, oligotrophic or eutrophic, the lake will be steered to; 
both policies are optimal. In the second case the threshold pollution level is a repelling equilibrium level; thus the optimal policy keeps that pollution level once started there, otherwise it steers the pollution level away from it.

Another important contribution of the present paper to the analysis of the shallow lake model is ascertained trade-off between social preferences and economic factors. It can be seen from the bifurcation diagrams with respect to the two parameters: $c$, relative costs of pollution, and $\rho$, the discount factor, see Figures 11-12. A decrease in $c$ may radically change the long run pollution level. In order to keep it at the same value the social planner has to become less myopic, $\rho$ has to be decreased proportionally.

\section{A Asymptotic behavior of the cusp curve in the parameter space}

In this Appendix we prove that the projections of the cusp bifurcation curve onto the $(b, c)-$ and $(b, \rho)$ - planes have the vertical asymptote $b=(\sqrt{9+6 \sqrt{3}}) / 8$ and its projection onto $(c, \rho)$-plane has the asymptote $\rho=K c+L$, where $K$ and $L$ are given by $(20)-(21)$.

The shallow lake system in state-control form is given by the following system of differential equations

$$
\left\{\begin{array}{l}
\dot{x}=u-b x+\frac{x^{2}}{x^{2}+1} \\
\dot{u}=-\left(\rho+b-\frac{2 x}{\left(x^{2}+1\right)^{2}}\right) u+2 c x u^{2} .
\end{array}\right.
$$

By solving the following system

$$
\left\{\begin{array}{l}
u-b x+\frac{x^{2}}{1+x^{2}}=0 \\
-\left(\rho+b-\frac{2 x}{\left(x^{2}+1\right)^{2}}\right) u+2 c x u^{2}=0
\end{array}\right.
$$

for $u>0$ we obtain the manifold of equilibria of the system (14) in the cartesian product $\mathbb{R} \times \mathbb{R}^{3}$ of state space and parameter space

$$
s(x ; b, c, \rho)=-\left(\rho+b-\frac{2 x}{\left(x^{2}+1\right)^{2}}\right)+2 c x\left(b x-\frac{x^{2}}{1+x^{2}}\right)=0 .
$$

From the definition of cusp bifurcation it follows that the cusp bifurcation curve is a solution of the following system

$$
\left\{\begin{array}{l}
s(x ; b, c, \rho)=0 \\
s_{x}(x ; b, c, \rho)=0 \\
s_{x x}(x ; b, c, \rho)=0 .
\end{array}\right.
$$

We want to solve the system (16) with respect to the parameters $b, c$ and $\rho$ to obtain an explicit expression for the cusp curve in the parameter space. For 
that we need to check solvability of (16) with respect to the parameters. The Jacobian of (16) is given by

$$
\frac{\partial\left(s, s_{x}, s_{x x}\right)}{\partial(b, c, \rho)}=-\frac{8 c x^{2}\left(-3+6 x^{2}+x^{4}\right)}{\left(1+x^{2}\right)^{3}} .
$$

For $c>0, x>0$ and $x \neq \sqrt{2 \sqrt{3}-3}$, which is the only positive root of the equation $-3+6 x^{2}+x^{4}=0$, the system (16) can be solved with respect to $b, c$ and $\rho$. Therefore the cusp bifurcation curve can be parameterized as the image of the map

$$
\Upsilon: x \mapsto\left(b_{\text {cusp }}(x), c_{\text {cusp }}(x), \rho_{\text {cusp }}(x)\right),
$$

where

$$
\begin{aligned}
b_{\text {cusp }}(x) & =\frac{-3 x-8 x^{3}+9 x^{5}+6 x^{7}}{\left(1+x^{2}\right)^{2}\left(-1-10 x^{2}+15 x^{4}\right)} \\
c_{\text {cusp }}(x) & =\frac{1+10 x^{2}-15 x^{4}}{x^{2}\left(1+x^{2}\right)\left(-3+6 x^{2}+x^{4}\right)} \\
\rho_{\text {cusp }}(x) & =\frac{2 x}{\left(1+x^{2}\right)^{3}}-\frac{5 x}{\left(1+x^{2}\right)^{2}}+\frac{15 x}{4\left(1+x^{2}\right)}-b_{\text {cusp }}(x)+\frac{x^{3}}{4} c_{\text {cusp }}(x)
\end{aligned}
$$

All the parameters in the model are assumed to be nonnegative; the inequalities $b_{\text {cusp }}(x) \geq 0, c_{\text {cusp }}>0$ and $\rho_{\text {cusp }} \geq 0$ imply that $x \in\left(\bar{x}_{1}, \bar{x}_{2}\right)$, where $\bar{x}_{1}=\sqrt{2 \sqrt{3}-3} \approx 0.68$, which is the root of the equation $3-6 x^{2}-x^{4}=0$, $\bar{x}_{2} \approx 0.8233$, which is a root of the equation $\rho_{\text {cusp }}=0$.

For $x \in\left(\bar{x}_{1}, \bar{x}_{2}\right)$ the functions $b_{\text {cusp }}(x), c_{\text {cusp }}(x)$ and $\rho_{\text {cusp }}(x)$ are monotone functions satisfying the following properties

$$
\begin{gathered}
b\left(\bar{x}_{1}\right)=\frac{1}{8} \sqrt{9+6 \sqrt{3}} \approx 0.5505, \\
\lim _{x \downarrow \bar{x}_{1}} c(x)=+\infty, \\
\lim _{x \downarrow \bar{x}_{1}} \rho(x)=+\infty .
\end{gathered}
$$

That proves that the projection of the cusp bifurcation curve $\Upsilon$ both on $(b, c)$ and $(b, \rho)$-planes has a vertical asymptote $b=(\sqrt{9+6 \sqrt{3}}) / 8$.

In order to prove that the projection of the cusp bifurcation curve on the $(c, \rho)$-plane has an inclined asymptote we compute the following limits

$$
\begin{aligned}
K & =\lim _{x \downarrow \bar{x}_{1}} \frac{\rho(x)}{c(x)}=\frac{1}{4}(2 \sqrt{3}-3)^{\frac{3}{2}}, \\
L & =\lim _{x \downarrow \bar{x}_{1}}(\rho(x)-K c(x))=\frac{1}{4} \sqrt{2 \sqrt{3}-3} .
\end{aligned}
$$

That proves that the cusp bifurcation curve has the asymptote in $(c, \rho)$-plane, given by

$$
\rho=K c+L .
$$




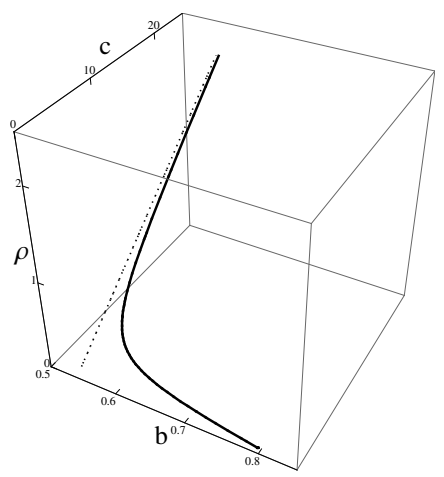

Figure 18: The cusp bifurcation curve and its asymptote in the parameter space $(b, c, \rho)$.

\section{B Asymptotic behavior of the saddle-node bi- furcation curves in the $(b, c)$-plane}

In this Appendix we prove that the saddle-node bifurcation curves in the $(b, c)$-plane have two vertical asymptotes $b=0$ and $b=1 / 2$.

We now write the shallow lake system in state-control form as

$$
\left\{\begin{array}{l}
\dot{x}=f(x, u ; b, c, \rho)=u-b x+\frac{x^{2}}{x^{2}+1} \\
\dot{u}=g(x, u ; b, c, \rho)=-\left(\rho+b-\frac{2 x}{\left(x^{2}+1\right)^{2}}\right) u+2 c x u^{2} .
\end{array}\right.
$$

Saddle-node bifurcations occur for points that are solutions of the following system

$$
\left\{\begin{array}{l}
f(x, u ; b, c, \rho)=0 \\
g(x, u ; b, c, \rho)=0 \\
D(x, u ; b, c, \rho)=0
\end{array}\right.
$$

where $D(x, u ; b, c, \rho)=\operatorname{det}(\partial(f, g) / \partial(x, u))$. It can be shown that the system (24) can always be solved with respect to the parameters $b, c$ and $\rho$ and the saddle-node bifurcation surface can be parameterized as image of the map

$$
\Gamma:(x, u) \mapsto\left(b_{s n}(x, u), c_{s n}(x, u), \rho_{s n}(x, u)\right) .
$$


Solving (24) yields

$$
\begin{aligned}
& b_{s n}(x, u)=\frac{x^{2}+u\left(1+x^{2}\right)}{x\left(1+x^{2}\right)} \\
& c_{s n}(x, u)=\frac{3 x^{2}-1}{\left(1+x^{2}\right)\left(2 u-x^{2}+4 u x^{2}+x^{4}+2 u x^{4}\right)} \\
& \rho_{s n}(x, u)=\frac{2 x}{\left(1+x^{2}\right)^{2}}+2 u x c_{s n}(x, u)-b_{s n}(x, u)
\end{aligned}
$$

Intersection of the saddle-node bifurcation surface with the plane $\rho=\rho_{0}$ can be computed by solving the following equation

$$
\rho_{s n}(x, u)=\rho_{0}
$$

with respect to $u$ for $\rho_{0}>0$. (28) is formally equivalent to a quadratic equation in $u$. Let us denote solutions of $(28)$ as $\hat{u}_{1}\left(x, \rho_{0}\right)$ and $\hat{u}_{2}\left(x, \rho_{0}\right)$. Then the intersection of the saddle-node surface $\Gamma(x, u)$ with the plane $\rho=\rho_{0}$ is defined as the two following curves

$$
\begin{aligned}
\gamma_{1}: x \mapsto \Gamma\left(x, \hat{u}_{1}\left(x, \rho_{0}\right)\right), \\
\gamma_{2}: x \mapsto \Gamma\left(x, \hat{u}_{2}\left(x, \rho_{0}\right)\right) .
\end{aligned}
$$

We want to determine the vertical asymptotes in the $(b, c)$-plane of the saddlenode curves $\gamma_{1}(x)$ and $\gamma_{2}(x)$, in other words we want to find $x^{*} \geq 0$ such that

$$
\begin{aligned}
\lim _{x \rightarrow x^{*}} b_{s n}\left(x, \hat{u}_{i}\left(x, \rho_{0}\right)\right) & =b^{*}<\infty, \\
\lim _{x \rightarrow x^{*}} c_{s n}\left(x, \hat{u}_{i}\left(x, \rho_{0}\right)\right) & =\infty, \\
\lim _{x \rightarrow x^{*}} \rho_{s n}\left(x, \hat{u}_{i}\left(x, \rho_{0}\right)\right) & =\rho_{0} .
\end{aligned}
$$

As $x$ converges to $x^{*} c_{s n}$ diverges to infinity, but $b_{s n}$ and $\rho_{s n}$ have finite limits; the equation (27) implies that either $x^{*}=0$ or $\lim _{x \rightarrow x^{*}} \hat{u}_{i}(x)=0$ or both together have to hold.

Let us consider the three possible cases:

- $x^{*}=0$ and $\lim _{x \rightarrow x^{*}} \hat{u}_{i}(x) \neq 0$

Together with (25) this implies that $\lim _{x \rightarrow^{*}} b_{s n}\left(x, \hat{u}_{i}\right)=\infty$, contradicting (29).

- $\lim _{x \rightarrow x^{*}} \hat{u}_{i}(x)=0$ and $x^{*}>0$

Together with (26) and (30) this implies that $x^{*}=1$. The solution of (28) for $x \approx x^{*}$ can be written as

- for $\rho_{0}<1 / 4$

$$
\begin{aligned}
& \hat{u}_{1}(x)=\left(\frac{1}{4}-\rho_{0}\right)-\left(\rho_{0}+\frac{1}{4\left(1-4 \rho_{0}\right)}\right)(x-1)+o\left((x-1)^{2}\right), \\
& \hat{u}_{2}(x)=\frac{\rho_{0}}{1-4 \rho_{0}}(x-1)+o\left((x-1)^{2}\right) .
\end{aligned}
$$


- for $\rho_{0}>1 / 4$

$$
\begin{aligned}
& \hat{u}_{1}(x)=\frac{\rho_{0}}{1-4 \rho_{0}}(x-1)+o\left((x-1)^{2}\right), \\
& \hat{u}_{2}(x)=\left(\frac{1}{4}-\rho_{0}\right)-\left(\rho_{0}+\frac{1}{4\left(1-4 \rho_{0}\right)}\right)(x-1)+o\left((x-1)^{2}\right) .
\end{aligned}
$$

For $\rho_{0}<1 / 4$

$$
\lim _{x \rightarrow x^{*}} \hat{u}_{1}(x)=1 / 4-\rho_{0}>0,
$$

contradicting the assumption. Hence we leave the solution $\hat{u}_{1}(x)$ out for $\rho_{0}<1 / 4$. This implies that

$$
\lim _{x \rightarrow x^{*}} b_{s n}\left(x, u_{2}(x)\right)=b_{s n}(1,0)=1 / 2 .
$$

For $\rho_{0}>1 / 4$

$$
\lim _{x \rightarrow x^{*}} \hat{u}_{2}(x)=1 / 4-\rho_{0}>0,
$$

contradicting the assumption. Hence we leave the solution $\hat{u}_{2}(x)$ out for $\rho_{0}>1 / 4$. This implies that

$$
\lim _{x \rightarrow x^{*}} b_{s n}\left(x, u_{1}(x)\right)=b_{s n}(1,0)=1 / 2 .
$$

For $\rho=1 / 4$

$$
\lim _{x \rightarrow x^{*}} \hat{u}_{1}(x)=\lim _{x \rightarrow x^{*}} \hat{u}_{2}(x)=0,
$$

implying that

$$
\lim _{x \rightarrow x^{*}} b_{s n}\left(x, u_{1}(x)\right)=\lim _{x \rightarrow x^{*}} b_{s n}\left(x, u_{2}(x)\right)=b_{s n}(1,0)=1 / 2 .
$$

- $\lim _{x \rightarrow x^{*}} \hat{u}_{i}(x)=0$ and $x^{*}=0$

The solutions of the equation (28) for $x \approx x^{*}$ can be written as

$$
\begin{aligned}
& \hat{u}_{1}\left(x, \rho_{0}\right)=\frac{1}{2} x^{2}-\frac{1}{2 \rho_{0}} x^{3}+o\left(x^{4}\right), \\
& \hat{u}_{2}\left(x, \rho_{0}\right)=-\rho_{0} x+\frac{1}{2 \rho_{0}} x^{3}+o\left(x^{4}\right) .
\end{aligned}
$$

Since $\lim _{x \rightarrow x^{*}} b_{s n}\left(x, \hat{u}_{2}(x)\right)=-\rho_{0}$ and since we do not allow the parameter $b$ to be negative, we are interested only in the solution $\hat{u}_{1}(x)$ which gives the second asymptote of the saddle-node bifurcation curve $\gamma_{1}(x)$ :

$$
\lim _{x \rightarrow x^{*}} b_{s n}\left(x, \hat{u}_{1}(x)\right)=0 .
$$

The analysis given above implies that the saddle-node bifurcation curves $\gamma_{i}(x)$ have the vertical asymptotes $b=0$ and $b=1 / 2$ for any $\rho_{0}>0$. Moreover, it can be proven that the saddle-node bifurcation curves have no other asymptotes in the $(b, c)$-plane. 


\section{References}

Brock, W.A., Starrett, D., 2003. Managing systems with non-convex positive feedback. Environmental and Resource Economics 26, 575-602.

Boltyanski, V.G., 1966. Sufficient conditions for optimality and justification of dynamic programming method. SIAM Journal of Control 4:2, 326-361.

Caulkins, J. P., Feichtinger, G., Grass, D., Tragler, G., 2007. Bifurcating DNS Thresholds in a Model of Organizational Bridge Building. Journal of Optimization Theory and Applications 133:1, 19-35.

Grass, D., Caulkins, J.P., Feichtinger, G., Tragler G., Behrens, D.A., 2008. Optimal Control of Nonlinear Processes. Springer.

Guckenheimer, J., Holmes, Ph., 1983. Nonlinear Oscillations, Dynamical Systems, and Bifurcations of Vector Fields. Spinger-Verlag.

Dieudonné, J., 2007. Éléments d'Anaylse. Tom I: Fondements de l'Analyse Moderne. Éditions Jacque Gabay.

Kiseleva, T., Wagener, F.O.O., in preparation. Bifurcations of optimal vector fields.

Kossioris, G., Plexousakis, M., Xepapadeas, A., de Zeeuw, A., Mäler, K.-G., 2008. Feedback Nash equilibria for non-linear differential games in pollution control. Journal of Economic Dynamics and Control, 32:4, 1312-1331.

Kuznetsov, Yu. A., 1998. Elements of Applied Bifurcation Theory. Spinger.

Mäler, K.-G., Xepapadeas, A., de Zeeuw, A., 2003. The economics of shallow lakes. Environmental and Resource Economics 26:4, 105-126.

Scheffer, M., 1998. Ecology of shallow lakes. Chapman\&Hall.

Steindl, A., Feichtinger, G., 2004. Bifurcations to Periodic Solutions in a Production/Inventory Model. Journal of Nonlinear Science 14:6, 469-503.

Wagener, F.O.O., 2009. Shallow lake economics run deep: Nonlinear aspects of an economic-ecological interest conflict. CeNDEF working paper,

http://www1.fee.uva.nl/cendef/publications/papers/Shallow_review.pdf

Wagener, F.O.O., 2003. Skiba points and heteroclinic bifurcations, with applications to the shallow lake system. Journal of Economic Dynamics and Control 27, 1533-1561.

Wagener, F.O.O., 2006. Skiba points for small discount rates. Journal of Optimization Theory and Applications 128:2, 261-277.

Wagener, F.O.O., 2005. Structural analysis of optimal investment for firms with non-concave production. Journal of Economic Behavior and Organization $57: 4,474-489$. 